\title{
Aspectos ecológicos e levantamento malacológico para identificação de áreas de risco para transmissão da esquistossomose mansoni no litoral norte de Pernambuco, Brasil
}

\author{
Marco A. A. Souza ${ }^{1,2}$, Verônica S. Barbosa ${ }^{2}$, Jones O. Albuquerque ${ }^{3}$, Silvana Bocanegra ${ }^{3}$, \\ Reinaldo Souza-Santos ${ }^{4}$, Helen Paredes ${ }^{4} \&$ Constança S. Barbosa ${ }^{2}$
}

1. Centro Universitário Norte do Espírito Santo, Departamento de Ciências da Saúde, Universidade Federal do Espírito Santo. Rodovia Br 101 Norte, km 60, Bairro Litorâneo, 29932-540 São Mateus, ES. (marcosouza@ceunes.ufes.br)

2. Centro de Pesquisas Aggeu Magalhães, Departamento de Parasitologia, Fundação Oswaldo Cruz. Avenida Moraes Rego, s/n, Caixa Postal 7472, Cidade Universitária, 50670-420 Recife, PE. (sbveronica85@hotmail.com; cbarbosa@cpqam.fiocruz.br)

3. Departamento de Estatística e Matemática, Universidade Federal Rural de Pernambuco. Rua Dom Manuel de Medeiros, s/n, Dois Irmãos, 52171-900 Recife, PE. (joa@ deinfo.ufrpe.br; silvana@ deinfo.ufrpe.br)

4. Escola Nacional de Saúde Pública, Departamento de Endemias Samuel Pessoa, Fundação Oswaldo Cruz. Rua Leopoldo Bulhões, 1470, Manguinhos, 21041-210 Rio de Janeiro,RJ. (rssantos@ensp.fiocruz.br; helenparedes@ensp.fiocruz.br)

\begin{abstract}
Ecological aspects and malacological survey to identification of transmission risk' sites for schistosomiasis in Pernambuco North Coast, Brazil. It was carried out a malacological survey at Carne de Vaca beach, Goiana municipality, Pernambuco North coast, between November 2006 and October 2007 in order to know the malacological fauna of this town and verify the natural, slightly or more modified conditions of mollusks sites by using an habitat diversity protocol analysis. From 5,912 mollusks collected, they belong to seven species and four families, as follows: 5,209 Biomphalaria glabrata (Say, 1818) specimens, 113 of Drepanotrema lucidum (Pfeiffer, 1839), 55 of Drepanotrema cimex (Moricand, 1837), 13 of Drepanotrema anatinum (Pfeiffer, 1839), 222 of Melanoides tuberculatus (Muller, 1774), 263 of Pomacea sp. and 37 of Physa marmorata Guilding, 1828. Among B. glabrata collected, 44 specimens were positive for Schistosoma mansoni Sambon, 1907 cercariae and 91 specimens were positive for other trematode larvae. One Pomacea sp. specimen was positive for trematode larvae. These data, spatially georeferenced, will be used for determination of risk' sites for schistosomiasis transmission at Carne de Vaca beach and furthermore, they will be used for computational simulations to determine the expansion process of schistosomiasis in state of Pernambuco.
\end{abstract}

KEYWORDS. Gastropoda, Schistosoma mansoni, Carne de Vaca beach, freshwater ecosystems.

RESUMO. Realizou-se levantamento malacológico na praia de Carne de Vaca, município de Goiana, litoral norte de Pernambuco, entre novembro de 2006 e outubro de 2007, com o objetivo de conhecer a fauna malacológica dessa localidade e verificar as condições naturais, pouco ou bastante alteradas das áreas de estudo através da aplicação de um protocolo de avaliação de diversidade de hábitats. Foram coletados 5.912 moluscos, representados por sete espécies e quatro famílias, dos quais, 5.209 exemplares de Biomphalaria glabrata (Say, 1818), 113 de Drepanotrema lucidum (Pfeiffer, 1839), 55 de Drepanotrema cimex (Moricand, 1837), 13 de Drepanotrema anatinum (Pfeiffer, 1839), 222 de Melanoides tuberculatus (Muller, 1774), 263 de Pomacea sp. e 37 de Physa marmorata Guilding, 1828. Entre os exemplares de B. glabrata coletados, 44 mostraram-se positivos para Schistosoma mansoni Sambon, 1907 e 91 mostraram-se positivos para outras larvas de trematódeos. Um exemplar de Pomacea sp. mostrou-se positivo para larva de trematódeo. Os dados obtidos, georreferenciados espacialmente, serão utilizados para a determinação das áreas de risco para a transmissão da esquistossomose na praia de Carne de Vaca, além de simulações computacionais para estudos de previsibilidade e comportamento do processo de expansão da esquistossomose no estado de Pernambuco.

PALAVRAS-CHAVE. Gastropoda, Schistosoma mansoni, Praia de Carne de Vaca, ambiente de água doce.

Representando um dos principais problemas de saúde pública no Brasil, estima-se que a esquistossomose, doença também conhecida como "xistose", "barriga d'água" ou "mal do caramujo", afete 4,6\% da população brasileira ( 8.000 .000 de indivíduos), sendo que no estado de Pernambuco são 1.262.000 (15,2\%) infectados (BARbosa et al., 1996; CARvalho et al., 1998; Katz \& Peixoto, 2000). A esquistossomose é endêmica nas zonas da mata, litoral e agreste, estando distribuída em 72 dos 185 municípios do estado de Pernambuco e as prevalências em algumas dessas localidades são superiores a $50 \%$ (FAVRE et al., 2001; FARIAS et al., 2007). A doença ocorre predominantemente em indivíduos de áreas rurais, mas desde a década de 1990, há registros de casos em populações de áreas litorâneas (GONÇALVES et al., 1991; BARBOSA et al., 1996;
ARAÚJo et al., 2007), em especial naquelas que apresentam problemas de saneamento básico.

A atuação humana desempenha papel preponderante na ampliação do território colonizado pelos hospedeiros do Schistosoma mansoni Sambon, 1907, sendo que a migração de populações tem contribuído para a expansão da esquistossomose para regiões em desenvolvimento. $\mathrm{O}$ estabelecimento da esquistossomose em uma determinada região dependerá das espécies de planorbídeos existentes, do grau de saneamento básico, das condições climáticas que possibilitem a realização das diversas fases do ciclo larvário do parasito e do contato da população humana com os criadouros de moluscos (Coelho, 1959; Michelson, 1987).

Entre os planorbídeos hospedeiros intermediários de $S$. mansoni, as duas principais espécies ocorrem no 
estado de Pernambuco. Segundo Paraense (1972), a espécie mais importante tanto pela amplitude de distribuição quanto pela eficiência na transmissão é Biomphalaria glabrata (Say, 1818). Indivíduos desta espécie são responsáveis atualmente pela quase totalidade dos focos da doença no litoral pernambucano (Barbosa et al., 2001; Barbosa \& Silva, 2006). Biomphalaria straminea (Dunker, 1848), apesar de ser encontrada em quase todas as bacias hidrográficas do Brasil e adaptada às variações climáticas, tem a sua infecção natural em Pernambuco verificada, predominantemente, na Zona da Mata (BARBosa et al., 1996). Especificamente em Goiana, município pernambucano inserido na bacia do rio Goiana, cujos principais afluentes são os rios Capibaribe Mirim e Tracunhaém (CPRH, 2001), como em grande parte dos municípios do Brasil, observa-se a ausência de saneamento básico, o que gera condições ideais para manutenção de criadouros de moluscos e ao desenvolvimento da esquistossomose. De fato, este quadro já vem sendo observado no litoral de Pernambuco desde a década de 1990, com os primeiros registros de esquistossomose na praia de Porto de Galinhas e na ilha de Itamaracá (BARBOSA et al., 1996, 2001). No ano de 2006, em inquérito epidemiológico realizado pelo Laboratório de Esquistossomose do Centro de Pesquisas Aggeu Magalhães (CPqAM) em Goiana, foram detectados casos humanos para S. mansoni na praia de Carne de Vaca (Constança S. Barbosa, com. pess.), até então sem registros de transmissão da doença. Além disso, alguns exemplares de $B$. glabrata capturados apresentaram-se positivos para cercárias de $S$. mansoni, o que motivou a realização deste estudo. Este trabalho tem como objetivo analisar aspectos ecológicos em locais com focos de B. glabrata relevantes para a identificação de áreas de risco para transmissão da esquistossomose no litoral norte de Pernambuco.

\section{MATERIAL E MÉTODOS}

O estudo foi realizado na praia de Carne de Vaca (Fig. 1), município de Goiana, Zona da Mata, Pernambuco, distante cerca de $70 \mathrm{~km}$ da cidade do Recife. A população estimada é de 1.600 habitantes e a principal fonte de renda é a pesca, praticada de forma artesanal, e a exploração do coco(IBGE, 2000). Oclima é tropical úmido, com temperaturas médias anuais em torno de $24^{\circ} \mathrm{C}$ nos meses de julho e agosto, períodos considerados menos quentes, e de $27{ }^{\circ} \mathrm{C}$ em fevereiro, considerado o mês mais quente (CPRH, 2001).
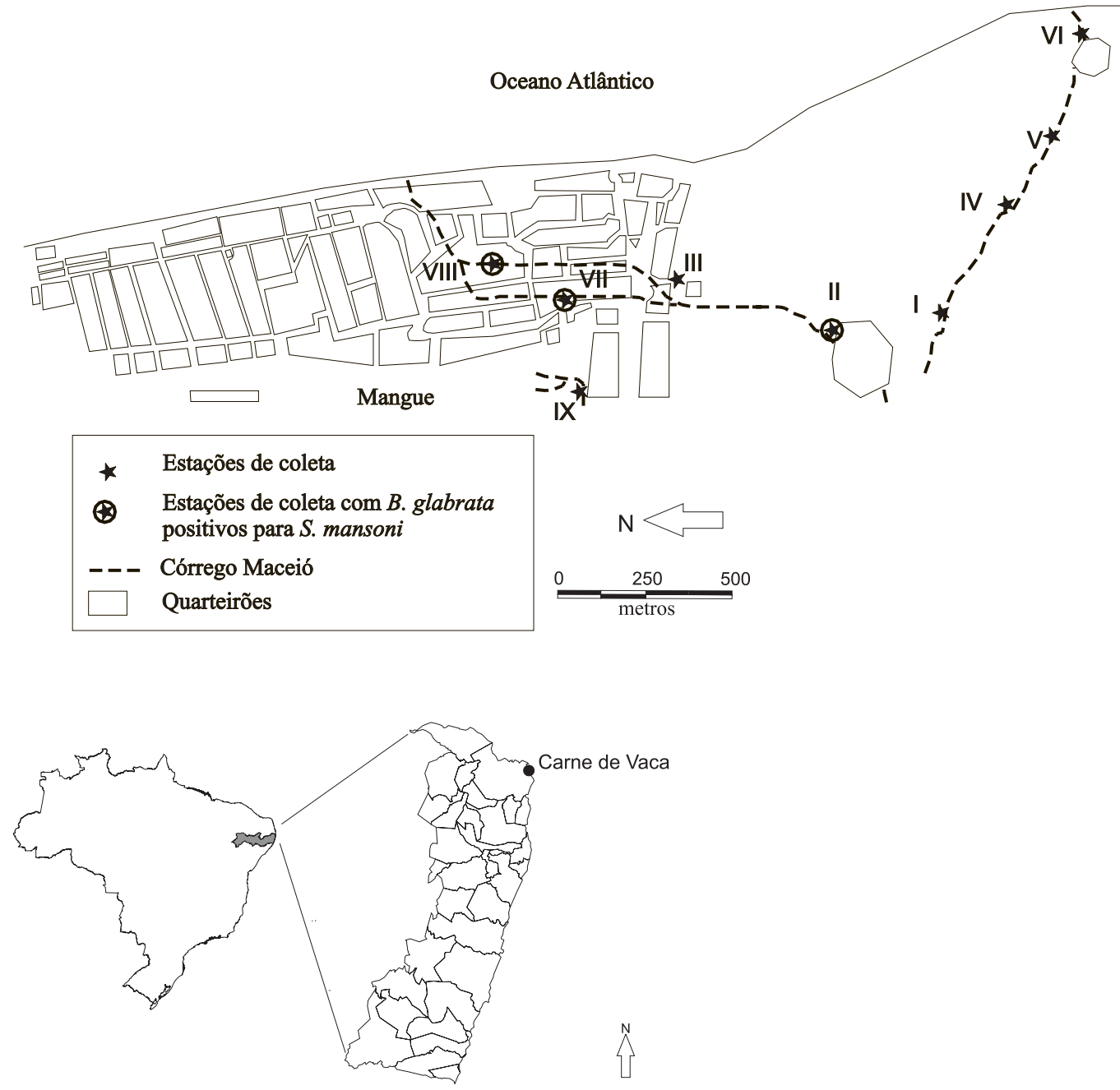

Figura 1. Croqui do distrito de Carne de Vaca, município de Goiana, Pernambuco, com as estações de coleta, assinalando a presença de moluscos Biomphalaria glabrata (Say, 1818) positivos para Schistosoma mansoni Sambon, 1907. 
Foram identificados os ambientes de água doce ocorrentes na localidade e respectivos sistemas lóticos, seus usos e condições de conservação, parâmetros estes utilizados para a seleção das estações de amostragem, cujas coordenadas geográficas foram obtidas através de GPS. Foi aplicado o protocolo de avaliação da diversidade de hábitats de acordo com CALLISTO et al. (2002) (Tab. I), que permite avaliar o impacto antrópico no ambiente aquático, através de uma pontuação específica para a área de estudo (GALDEAN et al., 2000), sendo uma importante ferramenta para o monitoramento ambiental (CALlisto et al., 2001).

Foram realizadas coletas mensais de um dia, entre novembro de 2006 e outubro de 2007, utilizando uma rede de mão (puçá), confeccionada com náilon $(50 \mathrm{~cm}$ de largura, $40 \mathrm{~cm}$ de altura, $30 \mathrm{~cm}$ de abertura, com malha de $\left.1 \mathrm{~mm}^{2}\right)$, adaptada a um cabo de madeira ou aço $(150 \mathrm{~cm}$ de comprimento). Foi aplicado um esforço amostral individual de 30 minutos, por varredura, em cerca de 10 (dez) metros de extensão, em cada uma das nove estações de coleta selecionadas (SouzA et al., 2006). Todo o material coletado por estação foi acondicionado em sacos plásticos, etiquetado e transportado para o Laboratório de Esquistossomose do Centro de Pesquisas Aggeu Magalhães (CPq AM), Recife, Pernambuco.

No laboratório, os moluscos foram triados, observados macroscopicamente para análise preliminar das espécies, contados, medidos o diâmetro das conchas e individualizados em pequenos recipientes de vidro "snapcap", com capacidade para $10 \mathrm{ml}$, contendo $5 \mathrm{ml}$ de água isenta de cloro. Em seguida, foram deixados durante a noite para exame antes e após fotoestimulação artificial direta (lâmpada de $60 \mathrm{~W}$ ), objetivando verificar a ocorrência de emergência de cercárias. No dia seguinte, após exame sem fotoestimulação, os mesmos foram examinados após expostos à luz por duas horas. Tal procedimento foi repetido semanalmente por 90 dias. Após este período, os moluscos que se mostraram negativos foram esmagados entre placas de vidro para a pesquisa de possíveis esporocistos, rédias e/ou metacercárias (CoutinHo, 1950).

De cada lote de planorbídeos coletados, foram retirados cerca de $10 \%$ de exemplares vivos e sacrificados em água a $70^{\circ} \mathrm{C}$. As partes moles foram fixadas em Railliet-
Henry e dissecadas sob estereomicroscópio (PARAENSE \& Deslandes, 1955). Para a identificação das espécies, foram considerados parâmetros conquiliológicos e morfológicos, seguindo MALEK (1962), PAHO (1968) e Paraense (1975). Os exemplares testemunhas foram incluídos na coleção do Laboratório do Serviço de Referência em Esquistossomose do CPqAM.

\section{RESULTADOS E DISCUSSÃO}

Foram estabelecidas nove estações de coleta nas coleções hídricas identificadas na localidade de Carne de Vaca, levando-se em consideração a presença humana e as alterações antrópicas (Fig. 1; Tab. II). A aplicação do protocolo de avaliação rápida da diversidade de hábitats ofereceu oportunidade para se avaliar os níveis de impactos antrópicos nos criadouros de planorbídeos identificados. Foram consideradas áreas altamente impactadas aquelas cuja pontuação atingiu até 20 pontos. Entre 20 e 36 pontos as áreas foram consideradas levemente impactadas e acima de 36 pontos foram consideradas em situação natural. Pela pontuação obtida (Fig. 2), verificou-se o predomínio de ambientes alterados na área de estudo, decorrentes de processos de ocupação desordenada do espaço urbano.

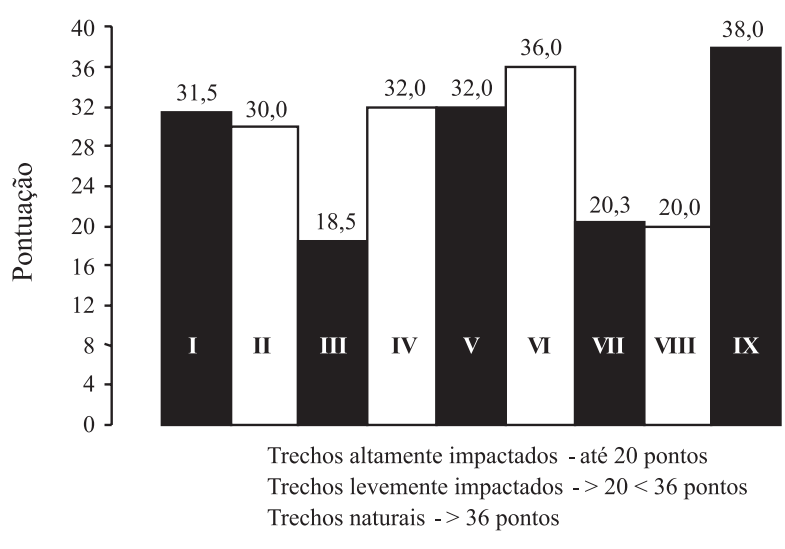

Figura 2. Avaliação rápida de diversidade de hábitats no distrito de Carne de Vaca, município de Goiana, Pernambuco, entre novembro de 2006 e outubro de 2007 (I a IX, estações de coleta).

Tabela I. Protocolo de avaliação rápida da diversidade de hábitats em trechos de bacias hidrográficas (CALLISTO et al., 2002) (4 pontos, situação natural; 2 e 0 pontos, situações pouco ou bastante alteradas).

\begin{tabular}{|c|c|c|c|}
\hline Parâmetros & 4 pontos & $\begin{array}{l}\text { Pontuação } \\
2 \text { pontos }\end{array}$ & 0 ponto \\
\hline $\begin{array}{l}\text { Tipo de ocupação das margens do } \\
\text { corpo d'água (principal atividade) }\end{array}$ & Vegetação natural & $\begin{array}{l}\text { Campo de pastagem/ Agricultura/ } \\
\text { Monocultura/ Reflorestamento }\end{array}$ & Residencial/ Comercial/ Industrial \\
\hline $\begin{array}{l}\text { Erosão próxima e/ ou nas margens } \\
\text { do rio e assoreamento em seu leito }\end{array}$ & Ausente & Moderada & Acentuada \\
\hline Alterações antrópicas & Ausentes & $\begin{array}{l}\text { Alterações de origem doméstica } \\
\text { (esgoto, lixo) }\end{array}$ & $\begin{array}{c}\text { Alterações de origem industrial/ } \\
\text { urbana (fábricas, siderurgias, canalização, } \\
\text { reutilização do curso do rio) }\end{array}$ \\
\hline Cobertura vegetal no leito & Parcial & Total & Ausente \\
\hline Odor da água & Nenhum & Esgoto (ovo podre) & Óleo/ Industrial \\
\hline Oleosidade da água & Ausente & Moderada & Abundante \\
\hline Transparência da água & Transparente & Turva/ cor de chá forte & Opaca ou colorida \\
\hline Odor do sedimento (fundo) & Nenhum & Esgoto (ovo podre) & Óleo/ Industrial \\
\hline Oleosidade do fundo & Ausente & Moderado & Abundante \\
\hline Tipo de fundo & Pedras/ cascalho & Lama/ areia & Cimento/ canalizado \\
\hline
\end{tabular}


Estes achados indicam que o estabelecimento e a manutenção das doenças endêmicas estão relacionados ao processo de degradação ambiental provocado pela ação humana, tal como observado por SouZA et al. (2006, 2008), em levantamentos malacológicos realizados nos municípios de Mariana, Minas Gerais, e Jaboatão dos Guararapes, Pernambuco. Da mesma forma, BARBosa et al. (2001), em Porto de Galinhas, também em Pernambuco, verificaram que um grande número de moradores e veranistas apresentaram infecção aguda para esquistossomose, após intensa exposição às cercárias de S. mansoni eliminadas pelos hospedeiros intermediários presentes nas coleções de água formadas nas principais vias de acesso dessa localidade durante o período anual das chuvas.

Das nove estações de coleta estabelecidas em Carne de Vaca, sete apresentaram graus distintos de alterações antrópicas (Fig. 2). Destacam-se como as mais relevantes (a) a ocupação residencial às margens do corpo d'água (levando a processos moderados e acentuados de erosão e assoreamento do leito), (b) as alterações de origem doméstica, tais como lixo e esgoto (uma importante fonte de matéria orgânica para desenvolvimento de moluscos) e (c) a presença de cobertura vegetal no leito (Nymphaea sp.), muito favorável à fixação de moluscos no ambiente de água doce.

Entre novembro de 2006 e outubro de 2007, foi coletado um total de 5.912 moluscos, dos quais 4.972 vivos e 940 conchas, representados por sete espécies pertencentes a quatro famílias (Planorbidae, Thiaridae, Ampullariidae e Physidae). Biomphalaria glabrata foi a espécie mais numerosa com 4.435 exemplares capturados vivos, dos quais $44(0,99 \%)$ mostraram-se positivos para cercárias de $S$. mansoni após fotoestimulação direta (Tab. III). A média do diâmetro de concha dos moluscos não infectados foi de $14 \mathrm{~mm}$ e de infectados, considerados adultos relativamente jovens, foi de $13 \mathrm{~mm}$, indicando se tratar de um processo ativo e recente de infecção desses hospedeiros intermediários. Por outro lado, a reduzida taxa de infecção dos exemplares de B. glabrata parasitados por $S$. mansoni pode ser explicada pelo fato de todos os moradores locais terem sido tratados com medicamentos específicos, tanto para esquistossomose quanto para outras parasitoses intestinais, entre setembro de 2006 e janeiro de 2007, após inquérito coproscópico realizado em ação conjunta entre a prefeitura de Goiana e o Centro de Pesquisas Aggeu Magalhães (CPqAM), em Recife (Constança S. Barbosa, com. pess.).

O encontro de exemplares de B. glabrata positivos para $S$. mansoni em Carne de Vaca, mesmo com baixa taxa de infecção, reforça e evidencia a expansão da esquistossomose para áreas litorâneas de Pernambuco, já registrada desde a década de 1990 com os primeiros casos de esquistossomose aguda na ilha de Itamaracá e em Porto de Galinhas. Antes restrita às zonas rurais e periferias de grandes cidades, a esquistossomose vem se dispersando nesse estado, acometendo moradores e veranistas de regiões litorâneas. Este fato é observado pelo encontro de moluscos hospedeiros intermediários de $S$. mansoni em areias utilizadas para a construção civil, uma vez que são trazidas do interior do estado e, em muitos casos, de regiões sabidamente endêmicas para a esquistossomose, estabelecendo novos criadouros de moluscos nessas áreas litorâneas (BARBOSA et al., 1996; BARbosa \& SiLva, 2006; AraúJo et al., 2007).

Em outros 91 (2,05\%) exemplares de B. glabrata capturados e em apenas um dos 219 exemplares de Pomacea sp., foram encontradas diversas formas larvais de trematódeos, em suas fases cercarianas, pertencentes às famílias Echinostomatidae, Strigeidae, Diplostomatidae e Spirorchiidae (Tab. III). É importante salientar que vários tipos de larvas emergentes de moluscos pertencentes às famílias Spirorchiidae e Diplostomatidae apresentam morfologia que à primeira observação, podem levar à confusão com as cercárias do parasito causador da esquistossomose, sendo muito importante o seu conhecimento, evitando-se, assim, falsos diagnósticos.

Há de se ressaltar também que o encontro de Physa marmorata Guilding, 1828, Drepanotrema lucidum (Pfeiffer, 1839), Drepanotrema cimex (Moricand, 1837), Drepanotrema anatinum (Pfeiffer, 1839) e Melanoides tuberculatus (Muller, 1774) representa o primeiro relato para o município. Com especial importância, os tiarídeos (M. tuberculatus) têm se dispersado muito e seu potencial reprodutivo e sua capacidade de colonizar vários tipos de hábitats, limita ou exclui certas espécies de pulmonados, particularmente aqueles relacionados à esquistossomose (Pointier \& McCullough, 1989).

Tabela II. Estações de coleta no distrito de Carne de Vaca, município de Goiana, Pernambuco, com as respectivas coordenadas geográficas e espécies de moluscos encontradas.

\begin{tabular}{|c|c|c|}
\hline Estações de coleta & Coordenadas geográficas & Espécies de moluscos encontradas \\
\hline EST I & $\mathrm{S} 07^{\circ} 35^{\prime} 33,5^{\prime \prime} \mathrm{W} 034^{\circ} 49^{\prime} 43,1^{\prime \prime}$ & ausentes \\
\hline EST II & $\mathrm{S} 07^{\circ} 35^{\prime} 30,8^{\prime \prime} \mathrm{W} 034^{\circ} 49^{\prime} 44,1^{\prime \prime}$ & $\begin{array}{c}\text { Biomphalaria glabrata, Drepanotrema lucidum, } \\
\text { Pomacea sp., Physa marmorata }\end{array}$ \\
\hline EST III & S $07^{\circ} 35^{\prime} 29,2^{\prime \prime}$ W $034^{\circ} 49^{\prime} 39,99^{\prime \prime}$ & $\begin{array}{c}\text { B. glabrata, D. lucidum, Drepanotrema cimex, } \\
\text { Drepanotrema anatinum, Melanoides tuberculatus, } \\
\text { Pomacea sp., P. marmorata }\end{array}$ \\
\hline EST IV & $\mathrm{S} 07^{\circ} 34^{\prime} 58,5^{\prime \prime} \mathrm{W} 034^{\circ} 50 \prime 08,3 "$ & ausentes \\
\hline EST V & S 07 34' 59, "1" W 034'50' 07,6" & ausentes \\
\hline EST VI & S $07^{\circ} 34^{\prime} 47,2^{\prime \prime}$ W $034^{\circ} 49^{\prime} 58,6^{\prime \prime}$ & ausentes \\
\hline EST VII & S $07^{\circ} 34^{\prime} 43,9^{\prime \prime}$ W $034^{\circ} 50$ ' 01,7 ', & $\begin{array}{c}\text { B. glabrata, D. lucidum, D. cimex, M. tuberculatus, Pomacea sp., } \\
\text { P. marmorata }\end{array}$ \\
\hline EST VIII & S $07^{\circ} 34^{\prime} 40,2^{\prime \prime}$ W 034 $50 ' 00,5^{\circ}$ & $\begin{array}{c}\text { B. glabrata, D. lucidum, D. cimex, D. anatinum, M. tuberculatus, } \\
\text { Pomacea sp., P. marmorata }\end{array}$ \\
\hline EST IX & $\mathrm{S} 07^{\circ} 34^{\prime} 54,5^{\prime \prime} \mathrm{W} 034^{\circ} 50^{\prime} 23,0^{\prime \prime}$ & ausentes \\
\hline
\end{tabular}


Tabela III. Moluscos coletados no distrito de Carne de Vaca, município de Goiana, Pernambuco, entre novembro de 2006 e outubro de 2007, destacando os positivos para Schistosoma mansoni Sambon, 1907 (*) e para outras formas larvais de trematódeos (**) (Echinostomatidae, Strigeidae, Diplostomatidae, Spirorchiidae).

\begin{tabular}{|c|c|c|c|c|c|}
\hline & Vivos & Mortos - conchas & Total & Positivos $*$ & Positivos $* *$ \\
\hline Biomphalaria glabrata (Say, 1818) & 4.435 & 774 & 5.209 & $44(0,99 \%)$ & $91(2,05 \%)$ \\
\hline Drepanotrema lucidum (Pfeiffer, 1839) & 66 & 47 & 113 & 0 & 0 \\
\hline D. cimex (Moricand, 1837) & 31 & 24 & 55 & 0 & 0 \\
\hline D. anatinum (Pfeiffer, 1839) & 9 & 4 & 13 & 0 & 0 \\
\hline Melanoides tuberculatus (Muller, 1774) & 192 & 30 & 222 & 0 & 0 \\
\hline Pomacea sp. & 219 & 44 & 263 & 0 & $1(0,46 \%)$ \\
\hline Physa marmorata Guilding, 1828 & 20 & 17 & 37 & 0 & 0 \\
\hline Total & 4.972 & 940 & 5.912 & 44 & 92 \\
\hline
\end{tabular}

Das quatro estações de coleta onde foram encontrados moluscos transmissores da esquistossomose, em três (II, VII e VIII) havia B. glabrata infectado (Fig. 1). Nota-se que dois desses locais (estações VII e VIII), situam-se no perímetro urbano e o terceiro deles (estação II), apesar de mais afastado, é de extrema relevância visto que grande parte da população o frequenta assiduamente para a coleta de água ou lavagem de roupa.

O encontro de $B$. glabrata como única espécie vetora da esquistossomose mansoni na praia de Carne de Vaca reforça sua importância epidemiológica como transmissora da doença no litoral pernambucano. Além disso, os resultados evidenciaram que das três estações consideradas como "impactadas" (III, VII, VIII) as duas últimas correspondem aos locais de encontro de exemplares de Biomphalaria positivos para S. mansoni. Nelas foram observadas a presença de dejetos humanos e lixo doméstico nas coleções hídricas, construções próximas às margens do corpo d'água, além de vegetação propícia à fixação e desenvolvimento de moluscos hospedeiros.

É importante ressaltar que nos quarteirões onde se encontram as estações VII e VIII existe fornecimento de água encanada, considerada por alguns autores como fator de proteção (CostA et al., 1994). Contudo, por estes mesmos quarteirões, passam pequenos córregos onde foram encontrados os exemplares de B. glabrata, o que pode significar risco para a população devido a sua proximidade com as habitações.

Não obstante, quando efetuada uma análise de características ecológicas, deve-se ter em mente possíveis variações ambientais que possam afetar a dinâmica epidemiológica do evento estudado. No caso de Carne de Vaca, verifica-se um regime de chuvas bem demarcado entre os meses de maio a agosto, quando se observa um aumento da lâmina d'água dos córregos que cortam o vilarejo e principalmente os dois quarteirões onde foram encontrados espécimes positivos, podendo, portanto, ser um veículo de dispersão dos hospedeiros intermediários.

Vale ressaltar que o aumento da lâmina d'água, que possibilita maior contato entre homem e este ambiente, está possivelmente relacionado às ações antrópicas produzidas durante o processo de ocupação desordenado. Tal hipótese surge a partir da constatação de mudanças ambientais que podem reduzir o escoamento de água dos córregos, semelhante ao ocorrido em Porto de Galinhas, também em Pernambuco (ARAújo et al., 2007).

Os resultados aqui encontrados descrevem um importante cenário da dinâmica da esquistossomose no litoral pernambucano, em áreas como as de Porto de Galinhas, Jaboatão dos Guararapes, Olinda, Ilha de Itamaracá, além de Carne de Vaca, onde a ação desordenada do homem proporciona o surgimento e manutenção de focos de Biomphalaria e perpetuação da esquistossomose (BArbosa et al., 1996, 2001; SouZA et al., 2008). Apesar disso, para melhor compreensão da dinâmica local, tornase necessário o uso de ferramentas mais elaboradas, tais como simuladores computacionais para análise de cenários e previsibilidade do processo de expansão da doença, tal como utilizado por ÁRAúJo et al. (2007).

Neste contexto, o cruzamento dos dados coletados com imagens de satélite fornecidas pelo GEOSERE (2008), considerando as características ambientais, tais como clima, relevo, vegetação e bacias hidrográficas, poderá ser realizado para tornar o modelo mais próximo da realidade. Além disso, o ANKOS (2007), desenvolvido especificamente para este propósito, surge como uma ferramenta capaz de automatizar as atividades, organizar e padronizar os dados, facilitar a recuperação e análise das informações, bem como a interface com outros sistemas. ANKOS (2007) também possui uma arquitetura totalmente modular e permite facilmente a integração com outros módulos que futuramente serão incorporados ao sistema, a fim de constituir a plataforma de simulação.

Agradecimentos. Aos técnicos da Fundação Nacional de Saúde, Sebastião Alves dos Santos e do Laboratório do Serviço de Referência em Esquistossomose do Centro de Pesquisas Aggeu Magalhães, Valdeci Francisco de Oliveira, pela colaboração fundamental nesta pesquisa.

\section{REFERÊNCIAS BIBLIOGRÁFICAS}

ANKOS. 2007. A New Kind of Simulator. Disponível em: <http://sourceforge.net/projects/ankos>. Acesso em: 09.01.2008. Araújo, K. C. G. M.; Resendes, A. P. C.; Souza-Santos, R.; Silveira Júnior, J. C. \& Barbosa, C. S. 2007. Análise espacial dos focos de Biomphalaria glabrata e de casos humanos de esquistossomose mansônica em Porto de Galinhas, Pernambuco, Brasil, no ano de 2000. Cadernos de Saúde Pública 23(2):409-417.

Barbosa, C. S.; Domingues, A. L. C.; Аbath, F.; Montenegro, S. M. L.; Guida, U.; Carneiro, J.; Tabosa, B.; Morais, C. N. L. \& Spinelli, V. 2001. Epidemia de esquistossomose aguda na praia de Porto de Galinhas, Pernambuco, Brasil. Cadernos de Saúde Pública 17(3):725-728.

Barbosa, C. S \& Silva, P. B. 2006. Aspectos físico-químicos e biológicos relacionados à ocorrência de Biomphalaria glabrata em focos litorâneos da esquistossomose em Pernambuco. Química Nova 29(5):901-906. 
Barbosa, C. S.; Silva, C. B. \& Barbosa, F. S. 1996. Esquistossomose: reprodução e expansão da endemia no Estado de Pernambuco no Brasil. Revista de Saúde Pública 30(6):609-616.

Callisto, M.; Ferreira, W. R.; Moreno, P.; Goulart, M. \& Petrúcio, M. 2002. Aplicação de um protocolo de avaliação rápida da diversidade de hábitats em atividades de ensino e pesquisa (MG-RJ). Acta Limnologica Brasiliensia 14(1):91-98.

Callisto, M.; Moreno, P. \& Barbosa, F. A. R. 2001. Habitat diversity and benthic functional trophic groups at Serra do Cipó, Southeast Brazil. Revista Brasileira de Biologia 61(2):259-266.

Carvalho, E. M. F.; Acioli, M. D.; Branco, M. A. F.; Costa, A. M.; Cesse, E. A. P.; Andrade, A. G. \& Mello, E. M. L. L. 1998. Evolução da esquistossomose na Zona da Mata Sul de Pernambuco. Epidemiologia e situação atual: controle ou descontrole? Cadernos de Saúde Pública 14(4):787-795.

Coelho, M. V. 1959. Distribuição geográfica da esquistossomose mansônica. Revista Brasileira de Malariologia e Doenças Tropicais 11:219-246.

Costa, M. F. L.; Rocha, R. S.; Magalhães, M. H. A. \& Katz, N. 1994. A hierarchical model for analysis of socio-economic variables and water contact patterns associated with the hepatosplenic form of schistosomiasis. Cadernos de Saúde Pública 10(2):241-253.

Coutinho, J. O. 1950. Índices de infestação natural dos planorbídeos pelas cercárias do Schistosoma mansoni na cidade do SalvadorBahia. Anais da Faculdade de Medicina de São Paulo 25:29-53.

CPRH (Agência Estadual do Meio Ambiente e Recursos Hídricos). 2001. Diagnóstico Sócio Ambiental do Litoral Norte de Pernambuco. Disponível em: <http://www.cprh.pe.gov.br>. Acesso em: 09.04.2007.

Farias, L. M. M.; Resendes, A. P. C.; Sabrosa, P. C. \& SouzaSAntos, R. 2007. Análise preliminar do Sistema de Informação do Programa de Controle da Esquistossomose no período de 1999 a 2003. Cadernos de Saúde Pública 23(1):235-239.

Favre, T. C.; Pieri, O. S.; Barbosa, C. S. \& Beck, L. 2001. Avaliação das ações de controle da esquistossomose implementadas entre 1977 e 1996 na área endêmica de Pernambuco, Brasil. Revista da Sociedade Brasileira de Medicina Tropical 34(6):569-576.

Galdean, N.; Callisto, M. \& Barbosa, F. A. R. 2000. Lotic ecosystems of Serra do Cipó, southeast Brazil: water quality and a tentative classification based on the benthic macroinvertebrates community. Aquatic Ecosystem Health \& Management 3(4):545-552.

GEOSERE. (Geoprocessamento e Sensoriamento Remoto). 2008. Universidade Federal Rural de Pernambuco. Disponível em: <http://www.dtr.ufrpe.br/geosere/>. Acesso em: 09.01.2008.

Gonçalves, J. F.; Santana, W.; Barbosa, C. S. \& Coutinho, A. 1991. Esquistossomose Aguda, de Caráter Episódico, na Ilha de Itamaracá, Estado de Pernambuco. Cadernos de Saúde Pública 7(3):424-425.

IBGE. (Instituto Brasileiro de Geografia e Estatística). Censo 2000. Disponível em: <http://www.ibge.gov.br/censo/>. Acesso em: 09.04.2007.

Katz, N. \& Peixoto, S. V. 2000. Análise crítica da estimativa do número de portadores de esquistossomose mansoni no Brasil. Revista da Sociedade Brasileira de Medicina Tropical 33(3):303-308.

MaleK, E. A. 1962. Laboratory Guide and Notes for Medical Malacology. Minneapolis, Burgess. 154p.

Michelson, E. H. 1987. The intermediate snail-host: An agenda for future study. Memórias do Instituto Oswaldo Cruz 82(4):193-195.

PAHO. (Pan American Health Organization). 1968. A guide for the identification of the snail intermediate hosts of Schistosomiasis in the Americas. Scientific Publication. n. $168,121 \mathrm{p}$.

Paraense, W. L. 1972. Fauna planorbídica do Brasil. In: Lacaz, C. S.; BaruzZi, G. R. \& Siqueira, J. R. W. eds. Introdução à Geografia Médica do Brasil. São Paulo, USP. p.213-239. 1975. Estado atual da sistemática dos planorbídeos brasileiros Arquivos do Museu Nacional do Rio de Janeiro 55:105-128.

Paraense, W. L. \& Deslandes, N. 1955. Observations on the morphology of Australorbis nigricans. Memórias do Instituto Oswaldo Cruz 53(1):121-124.

Pointier, J. P. \& McCullough, F. 1989. Biological control of the snail hosts of Schistosoma mansoni in the Caribbean area using Thiara sp. Acta Tropica 46(3):147-155.

Souza, M. A. A.; Barbosa, V. S.; Wanderlei, T. N. G. \& Barbosa, C. S. 2008. Criadouros de Biomphalaria, temporários e permanentes, em Jaboatão dos Guararapes, PE. Revista da Sociedade Brasileira de Medicina Tropical 41(3):252-256.

Souza, M. A. A.; Souza, L. A.; Machado-Coelho, G. L. L. \& Melo, A L. 2006. Levantamento malacológico e mapeamento das áreas de risco para transmissão da esquistossomose mansoni no município de Mariana, Minas Gerais, Brasil. Revista de Ciências Médicas e Biológicas 5(2):132-139.

Recebido em agosto de 2008. Aceito em agosto de 2009. ISSN 0073-4721

Artigo disponível em: www.scielo.br/isz 